\title{
New approaches in predicting and diagnosing preeclampsia: Congo Red Dot Paper Test (Review)
}

\author{
AIDA PETCA $^{1,2}$, RUXANDRA DIANA SINESCU $^{3,4}$, FLORICA SANDRU ${ }^{5,6}$, RAZVAN-COSMIN PETCA $^{7,8}$, \\ MIHAI CRISTIAN DUMITRASCU ${ }^{1,9}$, CLAUDIA MEHEDINTU ${ }^{1,10}$ and MONA ELENA ZVANCA ${ }^{1,2}$ \\ ${ }^{1}$ Deparment of Obstetrics and Gynecology, 'Carol Davila' University of Medicine and Pharmacy, 050474 Bucharest; \\ ${ }^{2}$ Department of Obstetrics and Gynecology, 'Elias' Emergency University Hospital, 011461 Bucharest; \\ ${ }^{3}$ Department of Plastic Surgery and Reconstructive Microsurgery, 'Carol Davila' University of Medicine and Pharmacy, \\ 050474 Bucharest; ${ }^{4}$ Department of Plastic Surgery and Reconstructive Microsurgery, 'Elias’ Emergency University Hospital, \\ 011461 Bucharest; ${ }^{5}$ Department of Dermatology, 'Carol Davila' University of Medicine and Pharmacy, 050474 Bucharest; \\ ${ }^{6}$ Department of Dermatology, 'Elias' Emergency University Hospital, 011461 Bucharest; ${ }^{7}$ Department of Urology, 'Carol \\ Davila' University of Medicine and Pharmacy, 050474 Bucharest; ${ }^{8}$ Department of Urology, 'Prof. Dr. Th. Burghele’ Clinical \\ Hospital, 050659 Bucharest; ${ }^{9}$ Department of Obstetrics and Gynecology, University Emergency Hospital, \\ 050098 Bucharest; ${ }^{10}$ Department of Obstetrics and Gynecology, 'Malaxa' Clinical Hospital, 022441 Bucharest, Romania
}

Received November 5, 2021; Accepted December 7, 2021

DOI: $10.3892 /$ etm.2022.11196

\begin{abstract}
Preeclampsia (PE), a complication of pregnancy that is characterized by de novo hypertension and proteinuria, remains a leading cause of morbidity and mortality during pregnancy, influencing $2.5-7 \%$ of singleton and $7-21 \%$ of twin pregnancies. At present, diagnosis is based on traditional but unreliable and nonspecific clinical markers, and treatment of PE is suboptimal, with minimal effect on maternal and fetal mortality and morbidity. With the hope of developing an affordable and simple procedure for PE prediction for developing countries, a previous study examined the use of Congo red staining of misfolded and damaged proteins in the urine of women with PE. This feature has diagnostic and prognostic potential since it precedes the onset of clinical manifestations
\end{abstract}

Correspondence to: Professor Ruxandra Diana Sinescu, Department of Plastic Surgery and Reconstructive Microsurgery, 'Elias' Emergency University Hospital, 17 Marasti Boulevard, 011461 Bucharest, Romania

E-mail: ruxandrasinescu@gmail.com

Abbreviations: BLK, blank sample; BMI, body mass index; CKD, chronic kidney disease; CR, Congo red; CRD, Congo red dot; CRR, Congo red retention; DR, detection rate; FPR, false positive rate; MAP, mean arterial pressure; MIDPE, medically indicated delivery for PE; PAPP-A, pregnancy-associated plasma protein-A; P-CRL, healthy pregnant controls; PE, preeclampsia; PIGF, placental growth factor; sENG, soluble endoglin; sFLT-1, soluble fms-like tyrosine kinase 1; sPE, severe pre-eclampsia; UTPI, uterine artery pulsatility index

Key words: Congo red, preeclampsia, congophilia, misfolded proteins, urinary test, fresh urinary sample and correlates with disease severity. The test is inexpensive, popular within the medical staff, easy to use, and identifies women with PE in only $3 \mathrm{~min}$. Obstetrical providers benefit from the Congo Red Dot Paper Test analysis, since a negative result promotes lesser waiting times in triage, prevents unneeded admissions, and diminishes the health costs associated per case.

\section{Contents}

1. Introduction

2. Current knowledge

3. Congo Red Dot (CRD) Paper Test

4. Technique

5. Clinical applicability

6. The latest study on singleton pregnancies

7. Conclusions

\section{Introduction}

During the last decades, ultrasound diagnosis had a remarkable effect on obstetrical practices and perinatal medicine, providing essential data for understanding morphological development and fetal physiology. It is difficult to imagine a fetal/obstetrical problem where ultrasound does not contribute to its solution (1). However, the solution to one of the most common obstetrical complications, preeclampsia (PE), remains unattainable.

PE, a pathologic entity of pregnancy defined by hypertension and proteinuria occurring after 20 weeks, remains a threatening complication that causes high morbidity and mortality, affecting, on average, $2.5-7 \%$ of singleton and $7-21 \%$ of twin pregnancies (2). 


\section{Current knowledge}

The pathophysiology of PE remains poorly elucidated, but the improper development of the placenta as early as the first trimester is widely considered a major etiological cause. Previous studies have revealed the involvement of endothelial dysfunction as a pathological contributor to PE (3-6) and increased load on the maternal circulation and heart (7). Most frequently, the clinical symptoms become evident in the late second and third trimester, manifesting as a combination of systemic changes, which mostly include hypertension, secondarily, renal insufficiency, and, in severe cases, liver failure. Whenever left untreated, PE may evolve to eclampsia, threatening maternal health and life by epilepsy such as seizures and stroke. The origins of PE are linked to the placenta and not to the fetus; thus, the simplest treatment for PE remains the delivery of the placenta (8). At present, what is known about the molecular pathology and the fundamental causes of PE remains scarce, and frequently the correct and prompt diagnosis is complicated by the heterogeneity of the symptoms. In this unclear context, all the resources worldwide have been directed to identify the actual pathophysiology of this disease and develop, if not a treatment, accurate prediction and prevention methods.

In the first decade of this millennium, the mystery surrounding the molecular pathogenesis of PE began to be unraveled with certain key discoveries concerning alterations in placental antiangiogenic factors and the misfolding proteins shaded by the villous syncytiotrophoblast (3).

The theories accepted to date by the practitioners sustain that PE emerges based on insufficient invasion of the trophoblast into the spiral arteries of the myometrium, a phenomenon defined as impaired placentation, leading to placental ischemia and fetal hypoxia, thus stimulating sustained endoplasmic reticulum and oxidative stress (9). The plasma of women diagnosed with PE has increased levels of antiangiogenic factors (sFLT-1 and sENG), endothelial activation markers such as cytokines, and adhesion molecules. Proteinuria, a criterion for diagnosis of PE, is considered to be caused by endothelial dysfunction (10). PE is subclassified into: i) Earlyonset PE (delivery at $34+0$ weeks); ii) pre-term PE (delivery at $<37+0$ weeks); iii) late-onset PE (delivery at $\geq 34+0$ weeks); and iv) term PE (delivery at $\geq 37+0$ weeks) (11).

The aforementioned classification is based only on gestational age. It carries great importance for obstetricians coping with preterm PE. They are challenged to decide the moment to terminate the pregnancy, equating the requirement of attaining in utero fetal maturation with the risks held by the mother and the fetus whilst continuing the pregnancy. At present, diagnosis is based on the knowledge of the clinical features of PE but is frequently nonspecific and unreliable; thus, the treatment of $\mathrm{PE}$ is delayed and even suboptimal, with very little impact on maternal and fetal mortality and morbidity.

Pursuing the early first-trimester diagnosis and prediction of $\mathrm{PE}$ is driven by the desire to have the opportunity to intervene in time, to improve the phenomenon of placentation, thus reducing the prevalence and the consequences of the disease.

The Fetal Medicine Foundation (https://fetalmedicine. org/) has revealed an algorithm for first-trimester aneuploidy screening, which has proven its value, also predicting PE in singleton pregnancies. The test combines two markers from the maternal serum, pregnancy-associated placental protein A (PAPP-A) and placental growth factor (PIGF) (ideally at 10-11 weeks of gestation), along with first-trimester maternal Doppler uterine artery pulsatility index (UTPI), mean arterial pressure (MAP) and prior obstetrical or non-obstetrical risk factors $(12,13)$. The statistical model of this algorithm foresees that for a false positive rate (FPR) of $10 \%$, the rate of detection (DR) will be as high as $96 \%$ for early PE and $77 \%$ for preterm PE, including early and intermediate cases (13-15). At a calculated risk on the first-trimester screening test equal to or more than 1 in 100, a woman is considered as high risk for developing PE. At this point, prophylactic measures consist of receiving aspirin, at a dose of $150 \mathrm{mg}$, starting at 11-14 +6 weeks of gestation (from the moment of the calculus), taken every night until 36 weeks of gestation, until delivery or at the moment when PE is diagnosed (12). The earlier the aspirin is started, the better the results in improving uterine flow (16).

Previous studies have revealed that the urine of preeclamptic women carries misfolded proteins (17-22). Mature proteins are shaped into a specific three-dimensional arrangement, defined as protein folding, a phenomenon that implies a compact structure derived from the nascent protein chain (23). The physiological function of a protein in a living cell is achievable only through folding (23). Factors such as the length of the protein, the number and amino acid sequence, the sub-structure of its parts, and the presence or absence of external pathological agents or intrinsically disordered regions have an impact on the protein folding $(24,25)$. The literature is diverse when defining a misfolded protein. The prefix 'mis-' signifies that something is inaccurate, flawed, or erroneous. The term 'misfolded' apart from the structural aspect implies a physiological and functional facet and must be acknowledged (23). An elementary definition of protein misfolding is a structurally aberrant state that loses its physiological activity (26). Pathophysiological changes in certain diseases can modify the proper folding of proteins and cause the structurally modified appearance of misfolded proteins. Protein misfolding is capable of participating in the pathogenesis of the disease through its intrinsic toxicity or secondarily with the reduced biological activity of the affected protein (27). It can also lead to aggregation, the amassing of two or more misfolded proteins that are associated in a dysfunctional process (28). To date, the misfolded proteins are acknowledged for their part in determining neurodegenerative disorders such as familial amyloidotic polyneuropathy, amyotrophic lateral sclerosis, Alzheimer's disease, Huntington's disease, spongiform encephalopathy, and Parkinson's, through the creation of amyloid plaques (27,29-31).

Observing the presence of altered proteins in the urine of preeclamptic women and hoping to discover an inexpensive and simple test for PE prediction for developing countries, Buhimschi et al (32) examined the use of Congo red (CR) coloring of modified proteins.

CR [the sodium salt of 3,3'-(1,1'-biphenyl-4,4'-diyl) bis (4-aminonaphthalene-1-sulfonic acid)] is an organic compound, water-soluble, first identified as a $\mathrm{pH}$ indicator, currently utilized for tinting sediments or atherosclerotic 
lesions and inflammation of blood vessels, urine or the cytoplasm (13), pioneered by Puchtler et al in 1962 (33).

Using an impartial large-scale study of proteins, Buhimschi et al observed that women with severe PE (sPE) necessitating medically indicated delivery for PE (MIDPE) exhibited a peculiar set of proteomic biomarkers, which included albumin and nonrandom proteoforms of SERPINA1 gene (34). This finding carried diagnostic and prognostic potential, a positive test preceding the beginning of clinical manifestations, and corresponded with the severity of the disease (34). A simple method was designed, whereby, urine that had been combined with CR was dripped on a baseless nitrocellulose sheet, which was then washed with increasing concentrations of solutions of methanol. Spots after testing urine from women with sPE, but not healthy pregnant controls (P-CRL), remained red after the methanol wash, demonstrating that women with PE exhibit urinary congophilia.

\section{CRD Paper Test}

It is long and well known that CR ligates the free hydroxyl groups through hydrogen bonds to cellulose fibers. Having a planar molecular configuration, $\mathrm{CR}$ is able to intervene between the cellulose fibers, thus explaining the ability to dye textiles irreversibly. The misfolded proteins containing an abundance of $\beta$-sheets have the same geometry as the cellulose fibers. The CR dripped on paper forms hydrogen bonds with cellulose, thus reducing the flow through the porous paper (a phenomenon which is called CR retardation), staining the surface with tight circles. In the context of PE, the urine sample contains aggregated and misfolded proteins that will react with $\mathrm{CR}$ proportionally with the concentration. When urine from women with PE is dripped on cellulose, only a small amount or no free $\mathrm{CR}$ will remain available to bind with cellulose fibers, and thus the aggregates stain as a wide pink circle. When all CR is blocked in amyloids, a homogenously pink circle appears, while whenever some free CR remains available to bind the cellulose fibers, the middle circle is still visible (35). The optimal paper to use for CR-urine solutions is the self-adhesive label, as it does not wrinkle when wet. It has been demonstrated that the three-fold interaction between CR, misfolded proteins in the urine of women suffering from PE, and cellulose fibers in the self-adhesive label paper, led scientists to design the CRD Paper Test with components provided either in bulk or as kit assemblies (35-38).

\section{Technique}

A total of $50 \mu \mathrm{l}$ of protein-normalized or non-normalized urine is combined with $1 \mu \mathrm{l}$ of CR solution ( $5 \mathrm{mg} / \mathrm{ml}$; cat no. C6277; Sigma-Aldrich, Merck KGaA). A blank sample (BLK) is obtained by mixing $0.5 \mu \mathrm{l}$ of CR stock solution with $25 \mu \mathrm{l}$ phosphate-buffered saline (PBS). The urine-CR mixtures are vortexed for $1 \mathrm{~h}$, after which $5 \mu \mathrm{l}$ of each combination is double spotted onto a nitrocellulose membrane (Pure Nitrocellulose Unsupported Transfer membrane $0.22 \mu \mathrm{m}$; Bio-Rad Laboratories, Inc.). With the aid of a transilluminator, the dots are spatially spread to correspond to the array format (35). For $\sim 15 \mathrm{~min}$, the array is left to dry after the solutions have been added. The following step is a water wash for $3 \mathrm{~min}$ succeeded by increasing concentrations of methanol [50\% methanol, 3 min; $70 \%$ methanol, 1 min; and 90\% methanol up until the time that the redness in the BLK probes washes away (10-15 min)]. In this process, the red color of the non-PE urine samples fades as the free CR colorant recedes. When urine is positive for misfolded proteins, the CR is attached by them on the paper test, and the dots remain evidently red. Images are observed before and post-methanol wash and transposed to grayscale. Lower quality of the nitrocellulose membranes often causes high BLK values [percentage of Congo red retention (CRR) $>10 \%$ ] (35).

The point-of-care test uses $150 \mu \mathrm{l}$ of fresh urine combined with the CR colorant. After $1 \mathrm{~min}$, four drops of this solution are added to the pre-prepared reaction paper. The result is observed at $3 \mathrm{~min}$ against the visual aid provided. The results are interpreted with a visual colorimetric scale marked as negative, weak positive, and strongly positive (35).

\section{Clinical applicability}

The main finding of the studies performed thus far, is that PE may be diagnosed without error using a CR test when signs and symptoms appear (39). For an FPR of $15.3 \%$, the test had a DR of $94.1 \%$ (14), but the results were obtained on a small cohort and without considering standard biochemical markers and UTPI (12). It can be a simple, inexpensive, and useful tool in PE cases aiding the physicians with patient management. In this regard, it may assume the same role in PE diagnosis when compared with the PlGF stick test and sFlt/PIGF ratio test. Both of these other tests vary depending on the cutoff value used, and the sensitivity markedly decreases after week 34 of gestation. The DR in the first trimester ranges between 33.3-38.1\% for early PE, 16.1-27.4\% for late PE, and 20-24.7\% for all PE cases at an FPR of 12.8-15.8\%; thus the success of the tests in the prediction of PE early in the first trimester is not robust (13).

In the cases with preexisting hypertension or proteinuria, McCarthy et al (9) stated that women suffering from PE and chronic kidney disease (CKD) without PE and nonpregnant women with lupus nephritis have increased urine congophilia levels when compared with healthy pregnant women. An increased CRR is not always a reliable test to distinguish these afflictions, thus further research is required to reveal the place of congophilia in everyday practice in this matter. Another disadvantage of this test and all the tests available to date is that they do not include any information concerning multiple pregnancies. Thus far, there is no routine screening or diagnostic laboratory marker available. Screening for PE in multiple pregnancies is an urgent and vital topic since the number of multiple pregnancies is increasing due to widespread assisted reproductive technologies. The mean maternal age is higher and more likely to be associated with maternal hypertension disorders.

\section{The latest studies on singleton pregnancies}

Rood et al in 2019, conducted a prospective cohort study on 346 pregnant women assessed for PE. CRD paper test was performed on fresh urine sampling along with other tests 
already demonstrated to help diagnose soluble fms-like tyrosine kinase 1 (sFLT-1) and PlGF (35).

CRD paper test was positive in 86 out of the 346 cases (25\%), with a confirmed final $28 \%$ of all cases with PE. The CR test surpassed the urine and serum markers with $86.7 \%$ accuracy, $80.2 \%$ sensitivity, $89.2 \%$ specificity, and a negative predictive value of $92.1 \%$ (35).

In addition to this, Rood et al revealed that the CRD paper test could differentiate between PE and PE imitators, resulting in a lower iatrogenic preterm delivery rate. The implementation of this test in day-to-day practice, in high-income as well as less developed countries, could be cost-effective by reducing non-required hospitalization days, expenses such as facility and laboratory bills, and medical care. Analyzing the number of patients discharged following their first hospitalization, almost 246 inpatient care days could be potentially saved using this PE detection method (35).

Exhibiting promising results, the authors concluded that the CRD paper test is a valuable tool of low technology requirements for fast identification of $\mathrm{PE}$ (35).

The latest results from a prospective diagnostic casecontrol study in Bangladesh and Mexico conducted by Bracken et al (40) were published in January 2021. The survey evaluated the success of a beta prototype test in identifying misfolded proteins in the urine of women suffering from PE. The study assessed urine congophilia in 409 subjects ( $n=204 \mathrm{PE} ; \mathrm{n}=205$ uncomplicated pregnancies). A total of $85 \%$ of the clinical cases (83/98) in Bangladesh and 48\% (51/106) in Mexico were positive, as the GV-005 tested negative in $81 \%$ of the clinical controls (79/98) in the Bangladeshi group and $77 \%$ of the clinical controls (82/107) in the Mexican group. The outcomes confirmed that urine congophilia was promptly diagnosed utilizing the lateral flow diagnostic beta prototype of the device, GV-005 (40).

\section{Conclusions}

There are sufficient reasons to consider implementing the CRD paper test into our clinical practice. The test is inexpensive, easy to perform, and reliable when diagnosing PE. More extensive clinical trials are required to validate the preliminary results, as well as special study groups, including multiple pregnancies.

\section{Acknowledgements}

Not applicable.

\section{Funding}

No funding was received.

\section{Availability of data and materials}

Not applicable.

\section{Authors' contributions}

AP, RDS and MEZ designed the review. FS, RCP, MCD and $\mathrm{CM}$ performed the literature research and drafted the manuscript. AP, RDS and MEZ substantially contributed to the conception of the study, as well as revised and edited the final manuscript. Data authentication is not applicable. All authors have read and agreed to the published version of the manuscript.

\section{Ethics approval and consent to participate}

Not applicable.

\section{Patient consent for publication}

Not applicable.

\section{Competing interests}

The authors declare that they have no competing interests.

\section{References}

1. Nicolson M and Fleming JEE (eds): Imaging and imagining the fetus: The development of obstetric ultrasound. 1st edition. John Hopkins Univeristy Press, Baltimore, 2013.

2. ACOG Committee on Obstetric Practice: ACOG practice bulletin. Diagnosis and management of preeclampsia and eclampsia. Number 33, January 2002. American college of obstetricians and gynecologists. Int J Gynaecol Obstet 77: 67-75, 2002.

3. Romero R, Nien JK, Espinoza J, Todem D, Fu W, Chung H, Kusanovic JP, Gotsch F, Erez O, Mazaki-Tovi S, et al: A longitudinal study of angiogenic (placental growth factor) and antiangiogenic (soluble endoglin and soluble vascular endothelial growth factor receptor-1) factors in normal pregnancy and patients destined to develop preeclampsia and deliver a small for gestational age neonate. J Matern Fetal Neonatal Med 21: 9-23, 2008.

4. Than NG, Romero R, Tarca AL, Kekesi KA, Xu Y, Xu Z, Juhasz K, Bhatti G, Leavitt RJ, Gelencser Z, et al: Integrated systems biology approach identifies novel maternal and placental pathways of preeclampsia. Front Immunol 9: 1661, 2018.

5. Turco MY, Gardner L, Kay RG, Hamilton RS, Prater M, Hollinshead MS, McWhinnie A, Esposito L, Fernando R, Skelton H, et al: Trophoblast organoids as a model for maternalfetal interactions during human placentation. Nature 564: 263-267, 2018.

6. Redman CW, Sargent IL and Staff AC: IFPA senior award lecture: Making sense of pre-eclampsia-two placental causes of preeclampsia? Placenta 35 (Suppl): S20-S25, 2014.

7. Osol G and Bernstein I: Preeclampsia and maternal cardiovascular disease: Consequence orpredisposition? J Vasc Res 51: 290-304, 2014.

8. Hypertension in pregnancy. Report of the American college of obstetricians and gynecologists' task force on hypertension in pregnancy. Obstet Gynecol 122: 1122-1131, 2013.

9. McCarthy FP, Adetoba A, Gill C, Bramham K, Bertolaccini M, Burton GJ, Girardi G, Seed PT, Poston L and Chappell LC: Urinary congophilia in women with hypertensive disorders of pregnancy and preexisting proteinuria or hypertension. Am J Obstet Gynecol 215: 464.e1-e7, 2016.

10. Aouache R, Biquard L, Vaiman D and Miralles F: Oxidative stress in preeclampsia and placental diseases. Int J Mol Sci 19: 1496, 2018.

11. Brown MA, Magee LA, Kenny LC, Karumanchi SA, McCarthy FP, Saito S, Hall DR, Warren CE, Adoyi G and Ishaku S; International Society for the Study of Hypertension in Pregnancy (ISSHP): The hypertensive disorders of pregnancy: ISSHP classification, diagnosis \& management recommendations for international practice. Pregnancy Hypertens 13: 291-310, 2018.

12. Francisco C, Wright D, Benkő Z, Syngelaki A and Nicolaides KH: Competing-risks model in screening for pre-eclampsia in twin pregnancy according to maternal factors and biomarkers at 11-13 weeks' gestation. Ultrasound Obstet Gynecol 50: 589-595, 2017. 
13. Sammar M, Syngelaki A, Sharabi-Nov A, Nicolaides K and Meiri H: Can staining of damaged proteins in urine effectively predict preeclampsia? Fetal Diagn Ther 41: 23-31, 2017.

14. Wright D, Syngelaki A, Akolekar R, Poon LC and Nicolaides K: Competing risks model in screening for preeclampsia by materna characteristics and medical history. Am J Obstet Gynecol 213 : 62.e1-62.e10, 2015.

15. Poon LC, Shennan A, Hyett JA, Kapur A, Hadar E, Divakar H, McAuliffe F, da Silva Costa F, von Dadelszen P, McIntyre HD, et al: The international federation of gynecology and obstetrics (FIGO) initiative on pre-eclampsia: A pragmatic guide for first-trimester screening and prevention. Int J Gynaecol Obstet 145 (Suppl 1): S1-S33, 2019.

16. Webster K, Fishburn S, Maresh M, Findlay SC and Chappell LC; Guideline Committee: Diagnosis and management of hypertension in pregnancy: Summary of updated NICE guidance. BMJ 366: 15119, 2019 .

17. Buhimschi CS, Norwitz ER, Funai E, Richman S, Guller S, Lockwood CJ and Buhimschi IA: Urinary angiogenic factors cluster hypertensive disorders and identify women with severe preeclampsia. Am J Obstet Gynecol 192: 734-741, 2005.

18. Millen KR, Buhimschi CS, Zhao G, Rood KM, Tabbah S and Buhimschi IA: Serum and urine thioflavin-T-enhanced fluorescence in severe preeclampsia. Hypertension 71: 1185-1192, 2018

19. Nagarajappa C, Rangappa SS, Suryanarayana R and Balakrishna S: Urinary congophilia in preeclampsia: Experience from a rural tertiary-care hospital in India. Pregnancy Hypertens 13: 83-86, 2018.

20. Tong M, Cheng SB, Chen Q, DeSousa J, Stone PR, James JL, Chamley LW and Sharma S: Aggregated transthyretin is specifically packaged into placental nano-vesicles in preeclampsia. Sci Rep 7: 6694, 2017.

21. Cater JH, Kumita JR, Zeineddine Abdallah R, Zhao G Bernardo-Gancedo A, Henry A, Winata W, Chi M, Grenyer BSF, Townsend ML, et al: Human pregnancy zone protein stabilizes misfolded proteins including preeclampsia- and Alzheimer'sassociated amyloid beta peptide. Proc Natl Acad Sci USA 116 6101-6110, 2019

22. Cheng SB, Nakashima A and Sharma S: Understanding preeclampsia using Alzheimer's etiology: An intriguing viewpoint. Am J Reprod Immunol 75: 372-381, 2016.

23. Olzscha H: Posttranslational modifications and proteinopathies: How guardians of the proteome are defeated. Biol Chem 400 895-915, 2019.

24. Balchin D, Hayer-Hartl M and Hartl FU: In vivo aspects of protein folding and quality control. Science 353, aac4354, 2016.

25. Akerfelt M, Morimoto RI and Sistonen L: Heat shock factors: Integrators of cell stress, development and lifespan. Nat Rev Mol Cell Biol 11: 545-555, 2010.

26. Uemura E, Niwa T, Minami S, Takemoto K, Fukuchi S, Machida K, Imataka H, Ueda T, Ota M and Taguchi H: Largescale aggregation analysis of eukaryotic proteins reveals an involvement of intrinsically disordered regions in protein folding. Sci Rep 8: 678, 2018.
27. Sweeney P, Park H, Baumann M, Dunlop J, Frydman J, Kopito R, McCampbell A, Leblanc G, Venkateswaran A, Nurmi A and Hodgson R: Protein misfolding in neurodegenerative diseases: Implications and strategies. Transl Neurodegener 6: 6, 2017.

28. Tanikawa C, Ueda K, Suzuki A, Iida A, Nakamura R, Atsuta N, Tohnai G, Sobue G, Saichi N, Momozawa Y, et al: Citrullination of RGG motifs in FET proteins by PAD4 regulates protein aggregation and ALS susceptibility. Cell Rep 22: 1473-1483, 2018.

29. Spurrier J, Shukla AK, McLinden K, Johnson K and Giniger E: Altered expression of the Cdk5 activator-like protein, Cdk5 $\alpha$, causes neurodegeneration, in part by accelerating the rate of aging. Dis Model Mech 11, dmm031161, 2018.

30. Vilchez D, Saez I and Dillin A: The role of protein clearance mechanisms in organismal ageing and age-related diseases. Nat Commun 5: 5659, 2014

31. Wright PE and Dyson HJ: Intrinsically disordered proteins in cellular signalling and regulation. Nat Rev Mol Cell Biol 16: $18-29,2015$

32. Buhimschi IA, Nayeri UA, Zhao G, Shook LL, Pensalfini A, Funai EF, Bernstein IM, Glabe CG and Buhimschi CS: Protein misfolding, congophilia, oligomerization, and defective amyloid processing in preeclampsia. Sci Transl Med 6: 245ra92, 2014

33. Puchtler H, Sweat F and Levine M: On the binding of Congo Red by amyloid. J Histochem Cytochem 10: 355-364, 1962.

34. Buhimschi IA, Zhao G, Funai EF, Harris N, Sasson IE, Bernstein IM, Saade GR and Buhimschi CS: Proteomic profiling of urine identifies specific fragments of SERPINA1 and albumin as biomarkers of preeclampsia. Am J Obstet Gynecol 199: 551. e1-e16, 2008

35. Rood KM, Buhimschi CS, Dible T, Webster S, Zhao G, Samuels P and Buhimschi IA: Congo Red Dot Paper Test for antenatal triage and rapid identification of preeclampsia. EClinicalMedicine 8: 47-56, 2019.

36. Majors CE, Smith CA, Natoli ME, Kundrod KA and RichardsKortum R: Point-of-care diagnostics to improve maternal and neonatal health in low-resource settings. Lab Chip 17: 3351-3387, 2017.

37. Buhimschi IA, Buhimschi CS, Tagare H, Choma M and Jonas S: Methods and compositions for detecting misfolded proteins. U.S Patent Number 10, 324, 094 Filed April 10, 2014, issued June 18, 2019.

38. Li XM, Liu XM, Xu J, Du J and Cuckle H: Late pregnancy screening for preeclampsia with a urinary point-of-care test for misfolded proteins. PLoS One 15: e0233214, 2020.

39. Gerasimova EM, Fedotov SA, Kachkin DV, Vashukova ES, Glotov AS, Chernoff YO and Rubel AA: Protein misfolding during pregnancy: New approaches to preeclampsia diagnostics. Int J Mol Sci 20: 6183, 2019.

40. Bracken H, Buhimschi IA, Rahman A, Smith PRS, Pervin J, Rouf S, Bousieguez M, López LG, Buhimschi CS, Easterling T and Winik off B: Congo red test for identification of preeclampsia: Results of a prospective diagnostic case-control study in Bangladesh and Mexico. EClinicalMedicine 31: 100678, 2021. 